\title{
A Review on Underwater Image Enhancement Using Deep Residual Framework
}

\author{
Prof. Anuja Phapale ${ }^{1}$, Atal Deshmukh², Keshav Katkar², Onkar Karale², Puja Kasture ${ }^{2}$ \\ ${ }^{1}$ Assistant Professor at Information Technology Department, AISSMS Institute of Information Technology, \\ Pune, Maharashtra, India
}

2B.E. Scholar, Information Technology Department, AISSMS Institute of Information Technology, Pune, Maharashtra, India

\begin{abstract}
Article Info

Volume 8, Issue 3

Page Number : 52-56

Publication Issue

May-June-2021

\section{Article History}

Accepted : 01 May 2021

Published : 06 May 2021

There are various factors such as absorption, refraction \& the phenomenon of scattering of light by particles suspended in water that are responsible for distorted colors, low contrast \& blurred details of original underwater images. The traditional approaches include pre-processing the image using a descattering algorithm. The super-resolution (SR) method is applied. But this method has limitation that major part of the high frequency information is lost during descattering. This paper comes up with a solution for underwater image enhancement using a deep residual framework. Firstly, the generation of synthetic underwater images takes place for which cycle-consistent adversarial networks (CycleGAN) is employed. Further, these synthetic underwater images are used as training data for convolution neural network models. Secondly, the introduction of very-deep super-resolution reconstruction model to underwater resolution applications is carried out. Using this, the underwater Resnet model is proposed. It acts as a residual learning model for underwater image enhancement operations. Furthermore, the training mode \& loss function are improved. Then, a multi-term loss function is formed which comprises of proposed edge difference loss \& mean squared error loss. An asynchronous training mode is also being proposed that improves the performance of the multi-term loss function. Lastly, the discussion of the impact of batch normalization takes place. After a comparative analysis \& underwater image enhancements, we can say that detailed enhancement performance \& color correction of these proposed methods are much efficient $\&$ superior to that of previous traditional methods \& deep learning models.
\end{abstract}

Keywords: Asynchronous training, edge difference loss, residual learning, underwater image enhancement 


\section{INTRODUCTION}

Remote-operated vehicles (ROVs) and vision-targeted Autonomous Underwater Vehicles (AUVs) have eventually impacted the exploration of marine life recently. For such vision-targeted explorations, clear underwater images are essential pre-requisites. Although, the raw underwater images that we have obtained currently have color distortion, low contrast, and blurred details as a result of absorption, refraction of light, and scattering of suspended particles.

The absorption of red light is higher, whereas the transmission rate of green and blue light is better which states that the absorption of light by water is selective. However, raw underwater images are mostly green or blue as compared to that of an in-air image. We can divide the scattering of light into two types: forward scattering and backward scattering. Forward scattering light usually contributes to the blurred texture features of underwater objects as it comes from the object.

Backward scattering segregates the underwater image and causes noise as the light is reflected back before reaching the target object.

These challenges bring obstacles to tasks like tracking, segmentation, and vision-based navigation system. Therefore, for underwater vision tasks like deep-sea exploration, underwater monitoring, underwater robots, etc, image restoration plays a very vital role. The reliability of underwater vision tasks is promoted by underwater image enhancement by increasing the underwater image color contrast and reduction in the degradation caused by attenuation and scattering.
Thus, to deal with these challenges, this paper has proposed an underwater image enhancement solution using a deep residual framework. Hence, we provide a residual learning-based framework which improves the underwater image enhancement performance \& aims to build a deeper network unlike other deep learning based underwater enhancement approaches that focus on the relation between weakly supervised learning and generative adversarial networks.

\section{LITERATURE SURVEY}

According to Yang Wang, this paper deals and brings to our notice another method in this arena. Underwater images will always goes through from color distortion and the quality of visibility decreases due to the light absorption and scattering. There are existing methods that utilize various assumptions to achieve efficient solutions for underwater image enhancement. However, these methods share the common limitation although in some cases of scenes the embraced assumptions may fail. To deal with this problem, this paper proposes an end-to-end framework for the enhancement of underwater images. In that a CNN-based network called UIE-Net is opted. The UIE-net is trained with two important tasks, color reparation and haze removal. This unified training approach has enabled the learning of a strong feature representation for both the tasks simultaneously. A pixel disrupting technique is used in the proposed learning system to better extract the inherent features in local patches, which greatly increases the convergent learning rate, speed and accuracy. To carry out the training of UIE-net, we have synthesized 200000 training images based on the actual underwater imaging model. UIE-net outperforms current methods on benchmark underwater images for cross-scenes, according to tests. Finally I would like to give my concluding 
opinions about this paper. In this paper, they have proposed a CNN-based framework called IE-Net for underwater image enhancement, which contains two subnetworks: CC-Net, HR-Net. CC-Net outputs color absorption coefficients within different channels, which is then to be provided as an output.

According to Peigen Luo, the restoration of image qualities at a higher resolution was used for an improved visual perception. Also, it has shown significant performance as far as relative contrast enhancement, colorfulness and visual quality of enhanced image are concerned. But it has avoided the techniques to reduce the issue of noise as seen in the output images of the existing algorithms of haze removal and it imbalances the color of the input image. For many computer vision applications there are really useful algorithms on fog removal. As per literature survey here we found that many of the actual researchers have not focused on many issues related. The existing methods have bypassed the techniques to reduce the issue of noise as seen in the output images of the existing algorithms of haze removal. Much power or intention has not taken the dark channel prior (DCP). The problem of the uneven brightness is also not considered by the researchers. It reduces the performance of the haze removal algorithms.

According to $\mathrm{H}$. Serikawa, this paper deals and brings to our notice another method in this segment using descattering and fusion. This paper shows concerns about super-resolving underwater images and descattering. As per the best of our knowledge, there are some SR reconstruction algorithms for underwater imaging. A preprocessing step is proposed for removing noise and simply applied SR to images. This method does not take into consideration the scatter effects, light absorption, or texture loss. Thus, we propose a novel scheme for underwater SR and descattering simultaneously in this article. In the next part, we have introduced the recent trends of descattering and super-resolution methods. It has shown significant improvement in image visibility and has efficiently segmented the acute leukemia images. Although, computational complexity is more due to over exposed images with noise in the input images along with the image features. Also, light scattering degrades the visibility and results in low contrast of the captured image. As a result, Images usually suffer from low contrast, non-uniform lighting, blur, and diminished color.

According to G. Wang, in this paper they want to tell some information about the deep residual framework and presented a generative model based on deep residual network for single image super-resolution of underwater images for use by independent or selfstanding underwater robots. They have also provided an adversarial training pipeline for learning SISR from data paired. In order to supervise the training, they have formulated an objective function that evaluates the perceptual quality of an image based on its global content, color, and local style information. Secondly, we present USR-248, a large-scale dataset of three sets of underwater images of 'high reaching' and 'low reaching' resolution. This dataset that is used is USR248 and it contains over $7 \mathrm{~K}$ paired instances in each set of data for supervised training of $2 \times, 4 \times$, or $8 \times$ SISR models. Furthermore, they have also validated the effectiveness of their proposed model through qualitative and quantitative experiments and compare the results with several state-of-the-art models' performances. In this paper they have also analyzed the practical feasibility for applications such as scene understanding and attention modeling in noisy visual conditions. The core element of this proposed model in this paper is that a fully convolutional deep block that is residual in nature, designed to learn 2 times interpolation in the RGB image space. They have also tried to denote this building block as Deep Residual Multiplier (DRM) as this DRM scales the input features' that are extracted from the image into spatial dimensions by a factor of two. The qualitative and 
quantitative results that I have personally understood from that paper suggests that the network SR-DRM and SRDRM-GAN provide good quality HR visualizations for LR image patches, which is useful in tracking fast-moving targets and understanding of underwater scenes. Therefore, Autonomous underwater vehicles and remotely operated underwater vehicles can use this to zoom in a particular region of interest for the sole purpose of obtaining a detailed and improved visual perception. That operational consideration for using such deep learning models in embedded robotic platforms is the computational complexity. The qualitative and quantitative calculations which we recommend that the proposed model can learn to repair image qualities at a higher resolution for an improved visual observation. In the future, we follow to improve its performance for $8 \times$ SISR. It is more efficient than testing at each pixel location; the real computational cost is still a little high. The future work for us will focus on improving the efficiency of the proposed approach by using a fully-CNN implementation. For many computer vision applications there are really useful algorithms on fog removal. As per literature survey here we found that many of the actual researchers have not focused on many issues related. The existing methods have bypassed the techniques to reduce the issue of noise as seen in the output images of the existing algorithms of haze removal. Much power or intention has not taken the dark channel prior (DCP). The problem of the uneven brightness is also not considered by the researchers. It reduces the performance of the haze removal algorithms. As finally as for the conclusion, in this paper, we understood that they have presented a fullyconvolutional deep residual network-based model for underwater image super resolution at two times, four time and eight times scales. They have also provided adversarial training pipelines driven by a multi modal objective function that is also generative in nature, which is designed for the sheer purpose of evaluation of the given image quality based on its content, color, and texture information. In addition, the said authors have also presented a large-scale dataset named USR248 which contains paired underwater images of various resolutions for supervised training of SISR models.

\section{III.PROPOSED WORK}

The challenges of underwater image enhancement are similar to that of super-resolution reconstruction. Compared to an in-air image, a raw underwater image's color is distorted. However, underwater images are blurred, and details, such as the edges of the underwater objects, are missing or lost. Thus, underwater image enhancement requires color deviation correction and detail restoration. Similarly, super-resolution reconstruction aims to restore images' details. After supplying training data for a powerful supervised learning model with CycleGAN, the very-deep super-resolution (VDSR) model [3] was introduced to the underwater image enhancement task.

The VDSR model has 20 convolution layers. Each convolution layer uses 3_3 size, with a stride of 1 and zero-padding with 1 pixel. Such parameter settings ensure that the resolution of the input image is identical to that of the output image. Except for the 1 st and the last layers, each three-channel image data as input, generates 64-channel feature maps, and transmits them to the main body of the network. The last layer is the reconstruction layer. It receives 64channel feature maps and outputs three-channel residual images. These residual images are further added to the input images that generate the restored images. When the VDSR model is used for superresolution reconstruction, the input image is a highresolution image generated by bicubic interpolation of a low-resolution image, such that the input image and the output image are the same size. 
Therefore, when the VDSR model is applied to underwater image restoration, the size of input and output images does not need to be adjusted, and neither does the network structure. Only appropriate training data are needed for the network to learn the difference between underwater and in-air images.

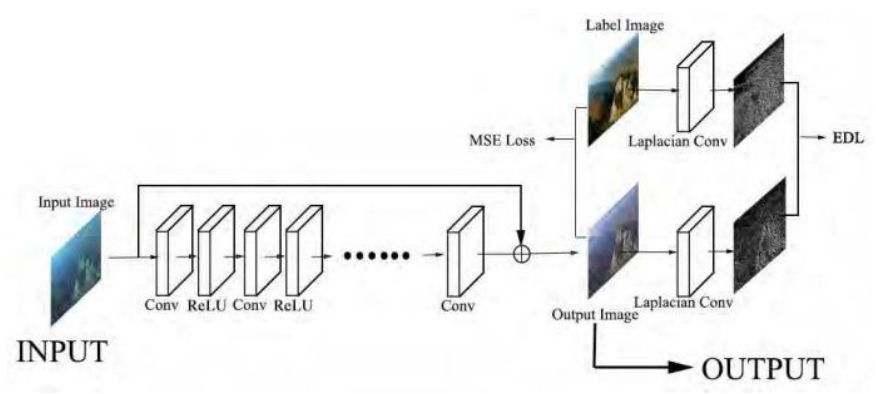

Figure 1: VDSR model with proposed EDL penalty term

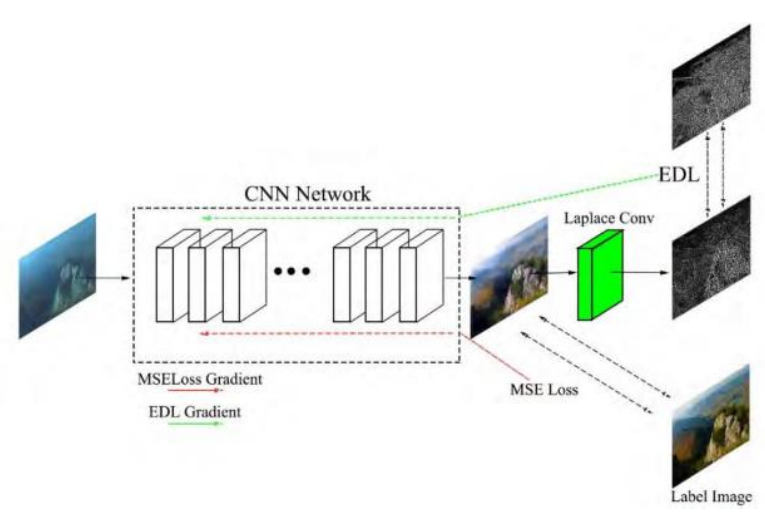

Figure 2: Proposed asynchronous training mode

\section{IV.OBJECTIVES}

- To recover a degraded image using a model of degradation and of the original image formation; is the main objective. These methods require multiple model parameters that are hardly available in tables and they can be extremely variable. Depth estimation of a given object in the scene is also an important parameter that is required.

- For producing a more visible image, the image enhancement technique uses qualitative subjective criteria and for image formation, they do not depend on any physical model.

- To enhance a highly turbid underwater image using the proposed framework.

- To obtain a visually pleasing result with better textures.

- To improve the capabilities of the existing system.

\section{v. CONCLUSION}

This paper has proposed an underwater image enhancement solution using a deep residual framework. Firstly, Cycle GAN was employed to generate synthetic underwater images as training data for the CNN models. Then, the super-resolution reconstruction model, i.e. the VDSR was introduced into the sector of underwater image enhancement, and further the residual learning model, Underwater Resnet (UResnet) was proposed. Furthermore, the loss function and training mode were improved; a multiterm loss function was formed with the proposed edge difference loss (EDL) and MSE loss indices. An asynchronous training mode has also been proposed that improves the performance of the multi-term loss function. The experimental results show the effectiveness of the proposed methods for underwater image restoration. EDL and the asynchronous training mode can improve the performances of CNN models on the underwater image enhancement task [10].

The proposed UResnet-P-A model achieved the best performance with regard to both color correction and detail enhancement than the other methods we compared, followed by the proposed UResnet and VDSR-P-A (BN) models. It has also been shown that BN layers, though harmful to super-resolution reconstruction, are helpful in the underwater image enhancement task. BN layers can accelerate convergence in training. Furthermore, the inclusion of BN layers can assist in further restoring details and enhancing image contrast. The proposed methods can efficiently improve the visual effects of underwater obtained images, which are helpful for the 
implementation of vision-based underwater tasks. These tasks include segmentation and tracking. Furthermore, we consider applying the proposed methods to the similar domains, such as image dehazing and super-resolution reconstruction to test the generality of the proposed methods. We leave these to our future work.

\section{REFERENCES}

[1] G. P.Liu, "Underwater Image enhancement using deep residual framework," 2019.

[2] H.Qi, "Underwater Image enhancement using deep residual framework," 2019.

[3] C.Zhenge, "Underwater Image enhancement using deep residual framework," 2019.

[4] Z.Yu, "Underwater Image enhancement using deep residual framework," 2019.

[5] P. Luo, "Underwater Image super-resolution using deep residual multipliers," 2019.

[6] Y. Wang, "A deep CNN Methood for the Underwater image enhancement," 2017.

[7] J. Sattar, "Underwater image super-resolution with deep residual multipliers," 2019.

[8] H. H.Lu, "Underwater Image super-resolution by descattering and fusion," 2017.

[9] R.Hummel, "Image enhancement by histogram transformation,Compute Graph,Image Process," 2017.

[10] S.Corchs, "Underwater Image processing. State of the art of restoration and image enhancement methods," 2014.

\section{Cite this article as :}

Prof. Anuja Phapale, Atal Deshmukh, Keshav Katkar,

Onkar Karale, Puja Kasture, " A Review on Underwater Image Enhancement Using Deep Residual Framework", International Journal of Scientific Research in Science and Technology (IJSRST), Online ISSN : 2395-602X, Print ISSN : 23956011, Volume 8 Issue 3, pp. 52-56, May-June 2021. Available at

doi : https://doi.org/10.32628/IJSRST218314

Journal URL : https://ijsrst.com/IJSRST218314 